\title{
FHigh Potential of Magnet on the Performance of Dual Piezoelectric Fans in Electronics Cooling System
}

\author{
Fadhilah Abdul Razak, Robiah Ahmad, Shamsul Sarip \\ UTM Razak School of Engineering and Advanced Technology, Universiti Teknologi Malaysia Kuala Lumpur/Razak \\ School UTMKL \\ Jalan Sultan Yahya Petra, 54100 Kuala Lumpur, Malaysia \\ Tel: +6(07)-5537599/37896/37894/SPS Razak School UTMKL
}

\begin{tabular}{l}
\hline \hline Article Info \\
\hline Article history: \\
Received Dec 4, 2017 \\
Revised Jan 28, 2018 \\
Accepted Feb 19, 2018 \\
\hline
\end{tabular}

Keywords:

Active cooling method

Heat transfer coefficient

Magnetic fans

Piezoelectric

Repulsive force

\begin{abstract}
Recently, piezoelectric fan has gained attention as potential active cooling method for electronics devices. Even though the piezoelectric requires high voltage, there are findings to overcome the shortcomings. Adding on a magnet at the tip of the piezoelectric fan to activate other magnetic passive fans is one of the methods to increase the total amplitude generated by the fans. This paper will discuss on the performance of integrated piezoelectric fan with passive fans (later refer to magnetic fans) to enhance the heat transfer in cooling system. A repulsive force produced by the magnets will cause the magnetic blades to oscillate together with the piezoelectric fan. The paper will focus on the optimization parameters of the magnets for selected dimension of piezoelectric fan. The parameters under investigation are the position of the magnet on the piezoelectric fan, number of magnets on each blades and orientation of blades with respect to adjacent blade. Results show that the magnet at middle location of extensive blade with double magnets generate the largest amplitude, $80 \%$ better than fan without magnet and for dual integrated piezoelectric fan with magnetic fan, radial orientation gives better result by $25 \%$. By increasing the total amplitude using magnetic force, power consumption can be reduced while the heat transfer performance can be enhanced. it shows a good agreement for positive heat transfer and thermal resistance improvement compared to natural convection.
\end{abstract}

Copyright $@ 2018$ Institute of Advanced Engineering and Science. All rights reserved.

\section{Corresponding Author:}

Fadhilah Abdul Razak, UTM Razak School of Engineering and Advanced Technology, Universiti Teknologi Malaysia Kuala Lumpur/Razak School UTMKL Jalan Sultan Yahya Petra, 54100 Kuala Lumpur, Malaysia. Tel: +6(07)-5537599/37896/37894/SPS Razak School UTMKL

Email: noordhilah@gmail.com

\section{INTRODUCTION}

The invention of piezoelectric fan is to replace current radial/axial fans [1] by reducing power consumption with noise free and long life. It is called a fan because the main purpose is to reduce temperature by increasing the air circulation in the area around the fan tip of the blade which are otherwise in stagnant state [2]. The piezoelectric fan is said to be long life because it is not using bearing as existed in radial/axial fans. Usage of ball bearing would degrade fan efficiency due to friction force [3]. Current rapid thermal management development of many machines causes air-cooling natural convection to be insufficient to encounter the heat dissipation from the electronic devices.

Heat transfer enhancement using piezoelectric fan falls under high amplitude vibratory motion of cooling technique [4]. The main concept is the oscillation of flexible piezoelectric fan will cause motion of air surrounding the blade/fan which results in improving heat transfer coefficient and heat transfer rates. An 
alternating input current (AC) causes the piezoelectric fan to contract and expand [5]. The oscillation of the fan causes the nearest air to the fan tip to move around with dynamic movement, depends on the behaviour of the flow field. The disturbing air helps to reduce the temperature in the system A fundamental piezoelectric fan is shown in Fig. 5. The oscillation of the blade will be at maximum when the frequency is at resonance [6]-[10] which is influenced by multiple factors. To further improve the blade oscillation, additional blade is attached to the piezoelectric fan, so that it is bending as a bimetallic strip [11].

A single piezoelectric fan will only affect a small area around the fan tip appropriate with small power consumption for each blade of the piezoelectric fan. Dual fans might enhance the heat transfer a bit thus multiple piezoelectric fans might expand the influence to a greater area. However, consuming many piezoelectric fans in a system require more cost as well. The fundamental heat transfer improvement using piezoelectric fan depends on the velocity and dispersion of air streams influenced by the piezoelectric blade [12]. Therefore, Yeom [13] in his study has undergone an experiment on multiple carbon fibre blades arranged in array driven by oval loop shell consist of piezoelectric stack actuator. The main aim is to gain maximum velocity and air stream induced to the surrounding air.

Different material to be attached to the piezoelectric blade would give different influence to the operating parameters such as resonance frequency and amplitude [2], [3]. Therefore, type of material attached to the piezoelectric blade plays important role in fabricating a high quality of piezoelectric fan. In the early stage of investigation, this aspect is not a vital focus thus the type of material was selected analytically such had been done by Acikalin by selecting stainless steel [14], phosphor copper by Su et al. [15] and Ma et al. [16]. Selection of suitable blade/beam involves few criteria such as light and flexible which require suitable density and Young Modulus of the material. By comparison done by Kimber et al. [2], Mylar gives the best performance of fan oscillation. By using Mylar, the result obtained for operating frequency is small with greater average amplitude which results in good air circulation with minimum power consumption [17].

Ma et al.[16] has come out with an idea of integration between a piezoelectric fan and several magnetic fans. The operation of the blades are driven by two forces; the vibration of the piezoelectric fan and repulsive magnetic force. The blades were arranged in an array orientation and it shows positive improvement in heat transfer performance. However, there is still less study on the significance of location of magnet on piezoelectric fan, number of magnets for each fan and orientation of multiple fans in radial orientation has been discovered. Therefore, this paper will be discussing on the said parameters.

\section{RESEARCH METHOD}

\subsection{Theoretical Analysis}

In a cooling system consisted of piezoelectric fan, one of the parameters that need to be optimized is the amplitude of the fan. With maximum amplitude achieved, the air flow velocity also will be enhanced thus disturbs the air flow surrounding the fan tip to reduce the temperature and enhance the heat transfer coefficient. A piezoelectric fan from piezo.com has been used throughout this study and the specifications as stated in Table 1. Table 2 shown accumulates the advantages of piezoelectric fan. Figure 1 shown piezoelectric fan by www.piezo.com

Table 1. Fan Specifications

\begin{tabular}{llll}
\hline Item & Description & Item & Description \\
\hline Input voltage & $115 \mathrm{VAC}, 60 \mathrm{~Hz}$ & Capacitance & $15 \mathrm{nF}$ \\
Power consumption & $30 \mathrm{~mW}$ & Volume flow rate & $2 \mathrm{CFM}(0.9 \mathrm{l} / \mathrm{s})$ \\
Peak air velocity & $400 \mathrm{FPM},(2.0 \mathrm{~m} / \mathrm{s})$ & Weight & 2.8 grams \\
Mounting & $\# 2-56 \mathrm{clr}$ holes, 2 places & Temperature range & $-20^{\circ} \mathrm{C}$ to $70^{\circ} \mathrm{C}$ \\
\hline
\end{tabular}

Table 2. Different Case Applied on Piezoelectric Fan and Magnetic Fans

\begin{tabular}{cccc}
\hline Case & No.of fan & No of magnet & Distance between magnets,mm \\
\hline 1 & 1 & 0 & 0 \\
2 & 1 & 1 & 0 \\
3 & 1 & 2 & 0 \\
3 & 2 & 1 & 15 \\
4 & 2 & 1 & 30 \\
5 & 2 & 1 & 40 \\
6 & 2 & 2 & 15 \\
7 & 2 & 2 & 30 \\
8 & 2 & 2 & 40 \\
\hline
\end{tabular}




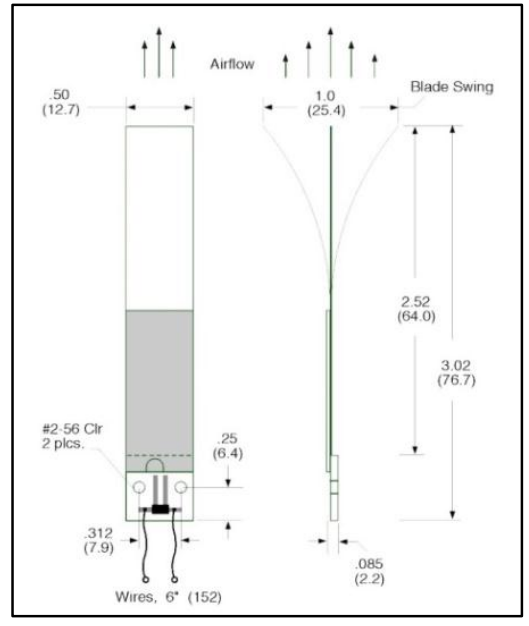

Figure 1. Piezoelectric Fan by www.piezo.com

The amplitude of the piezoelectric fan can be calculated using Equation1

$$
A=\frac{F L_{p f}^{3}}{3 E I}
$$

where $F, L_{p f}, E$ and $I$ are driving force, total length of the fan, Young Modulus of the piezoelectric fan and moment of inertia respectively. The driving force of the integrated piezoelectric fan magnetic fans originated from the vibration of the piezoelectric fan and repulsive magnetic force.

$$
\begin{aligned}
& F=\frac{3 w_{p f} t_{p f} d_{31} V E}{2 L_{p f}} \\
& F_{m a g}(x)=\left[\frac{B_{0}^{2} A_{m a g}^{2}\left(t_{m a g}^{2}+r_{m a g}^{2}\right)}{\pi \mu_{0} t_{m a g}^{2}}\right]\left[\frac{1}{x^{2}}+\frac{1}{\left(x+2 t_{m a g}\right)^{2}}-\frac{2}{\left(x+t_{m a g}\right)^{2}}\right]
\end{aligned}
$$

where $V, t_{p f}, d_{3 l}, E, L_{p f}$ and $w_{p f}$ are the applied voltage, thickness of the piezoelectric fan, the transverse piezoelectric constant, the Young's modulus of the piezoelectric fan, length and width of the fan respectively. The maximum amplitude occurred at effective resonance frequency, $\omega_{\text {eff }}$ of the piezoelectric fan. The resonance frequency can be calculated as in Equation 4.

$$
\omega_{e f f}=\frac{1}{2 \pi} \sqrt{\frac{K_{p f}+\Delta K_{m a g}}{m_{m a g}+0.23 m_{p f}}}
$$

where K_pf and K_mag are the stiffness for piezoelectric fan and magnetic fans respectively. The stiffness of the magnetic fan and piezoelectric fan can be found in Equation 3 and Equation 4:

$$
\begin{aligned}
& \Delta k_{m a g}(d)= \pm\left[\frac{B_{0}^{2} \pi r_{m a g}^{4}\left(t_{m a g}+r_{m a g}\right)^{2}}{\mu_{0} t_{m a g}^{2}}\right]\left|\left(-\frac{2}{d^{3}}-\frac{2}{\left(d+2 t_{m a g}\right)^{3}}+\frac{4}{\left(d+t_{m a g}\right)^{3}}\right)\right| \\
& k_{p f}=\frac{E w t_{p f}^{3}}{4 L_{p f}{ }^{3}}
\end{aligned}
$$

where $B_{0}, t_{m a g}, r_{m a g}, x$ and $\mu_{0}$ are the magnetic flux density, thickness of the magnet, radius of the magnet, distance between two magnets and permeability of the intervening medium respectively. At maximum amplitude, the integrated piezoelectric fan also encountered maximum air disturbance velocity which will affect the cooling rate of the cooling system. The velocity of the beam with respect to time is given in Equation 7 [18]: 


$$
v(x, t)=A \cdot\left[\begin{array}{c}
\left(\sin \left(\beta l_{u}\right)-\sinh \left(\beta l_{u}\right)\right)(\sin (\beta x)-\sinh (\beta x)) \\
+\left(\cos \left(\beta l_{u}\right)-\cosh \left(\beta l_{u}\right)\right)(\cos (\beta x)-\cosh (\beta x))
\end{array}\right] \cdot \omega_{b} \cos \left(\omega_{b} t\right)
$$

where $\beta$ values can be obtained from frequency equation as simplified in Equation 8:

$$
\begin{aligned}
& \cos \left(\beta l_{u}\right) \cdot \cos h\left(\beta l_{u}\right)=-1 \\
& \beta=\frac{1.875}{l_{u}}
\end{aligned}
$$

Position of additional mass such as magnet is very important in order to obtain largest amplitude with same input voltage. Based on Equation 1 maximum amplitude can be achieved at point of moment of inertia of the beam, I, where illustration is in Figure 2.

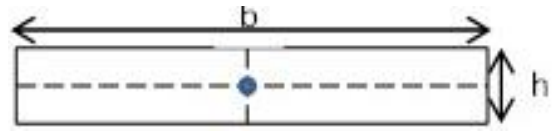

Figure 2 Moment of Inertia of a Beam (piezoelectric fan)

Moment of inertia is a measure of how difficult it is to rotate a particular body about a given axis. Greater the mass concentrated away from the axis, greater the moment of inertia [18]. In other words, moment of inertia is at the lowest value at the point on the axis of rotation to produce largest amplitude of the beam.

\subsection{Experimental Setup}

The experimental setup to find the maximum amplitude is started firstly by testing piezoelectric fan for calibration. Then the single fan is tested to get the best magnet location on the piezoelectric fan. Secondly, the number of magnets attached to the fan is varied, either single magnet or double magnets. Thirdly, dual fans system is tested for array and radial orientation by varying the distance between magnets.

By using specifications in Table 1 and Figure 1, a function generator is used to power up the piezoelectric fan. The function generator is able to control the current flow thus able to oscillate the piezoelectric at its resonant frequency. The piezoelectric needs 115VAC thus a step down transformer has been used to drop the voltage from 240 VAC to 115 VAC. For a dual fans system, 2 casings are produced which are in different orientation, array and radial respectively. The schematics are shown in Figure 3.

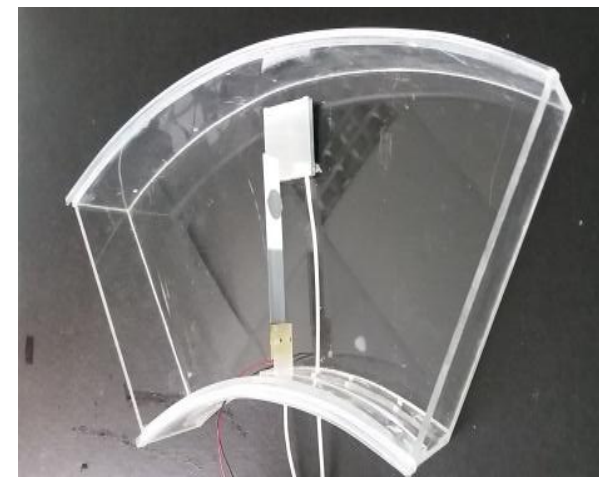

(a)

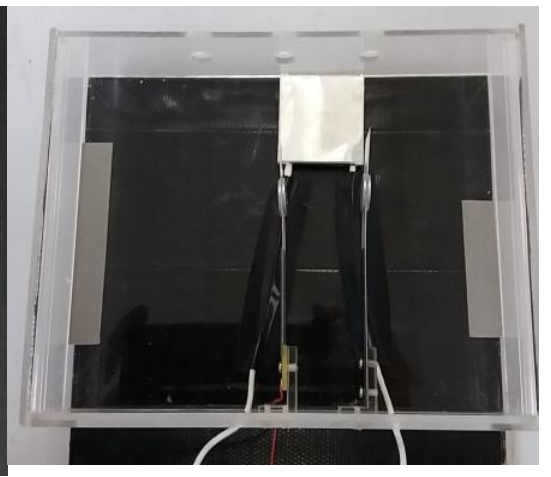

(b)

Figure 3. Orientation of Dual Piezoelectric Fan in (a)Radial and (b)Array Orientation 


\subsection{Experimental Procedure}

The overall experiment is about to prove the significant of magnet on piezoelectric fan, optimum number of magnet located on each fans and the optimum distance between magnets on the piezoelectric fan and adjacent passive fan. The radius of the magnet is kept constant as the position and distance between magnets are varied. The best position of the magnet was tested at single fan only with single magnet and dual magnet at position I, II and III as shown in Figure 4. The position of magnet with largest amplitude is selected for next experiment. Table 2 shown different cases applied on piezoelectric fan and magnetic fans.

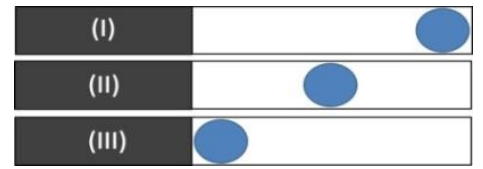

Figure 4. Location of Magnet on Piezoelectric Fan

Table 3. Different Case Applied on Piezoelectric Fan and Magnetic Fans

\begin{tabular}{cccc}
\hline Case & $\begin{array}{c}\text { No.of } \\
\text { fan }\end{array}$ & $\begin{array}{c}\text { No of } \\
\text { magnet }\end{array}$ & $\begin{array}{c}\text { Distance } \\
\text { between } \\
\text { magnets,mm }\end{array}$ \\
\hline 1 & 1 & 0 & 0 \\
2 & 1 & 1 & 0 \\
3 & 1 & 2 & 0 \\
3 & 2 & 1 & 15 \\
4 & 2 & 1 & 30 \\
5 & 2 & 1 & 40 \\
6 & 2 & 2 & 15 \\
7 & 2 & 2 & 30 \\
8 & 2 & 2 & 40 \\
\hline
\end{tabular}

\subsection{Performance Parameters}

The performance of the DPF system can be analyzed by calculating its enhancement in heat convection coefficient since it is related to temperature change. The heat flux, $q^{\circ}=\frac{Q^{\circ}}{A_{m c}}$, where equivalent to the ratio of power input and heated surface area. The heat convection coefficient can be measured as,

$$
h=\frac{q^{\circ}}{T_{s}-T_{a}}
$$

where $T_{s}$ is the average temperature of heat source and $T_{a}$ is the ambient temperature. Low thermal resistances are to be favored in nearly all categories of electronic packaging. Therefore, thermal resistance of the DPF system is,

$$
R_{t h(D P F)}=\frac{T_{s}-T_{a}}{h A}
$$

where A is the area of the heat source. The enhancement ratio of the system from natural convection to DPF cooling can be found as

$$
\varepsilon=\frac{h_{D P F}}{h_{n}}
$$

The type air flow generated by DPF cooling can be measured using Reynolds number, Re.

$$
R e=\frac{f_{r} A D_{p}}{v}
$$

The ratio of heat transfer through convection can be described by Nusselt number, $\mathrm{Nu}$, 


$$
N u=\frac{h D_{p}}{k}
$$

where $k$ is thermal conductivity of the air.

\section{RESULTS AND ANALYSIS}

\subsection{Significant of Magnet and its Location on Piezoelectric Fan}

A piezoelectric fan oscillated with larger amplitude when additional mass is located on the blade. The position of the mass is varied to three positions and at each position, single and double magnets are applied. A table of cases is summarized as shown in Table 4.

Table 4. Variety of Cases under Different Position of Magnet and Number of Magnet

\begin{tabular}{cccc}
\hline Case number & Magnet position & $\mathrm{f}_{\text {res }}(\mathrm{Hz})$ & Amplitude, $\mathrm{mm}$ \\
\hline No magnet & - & 58.39 & 12.5 \\
Single & I & 16.2 & 11 \\
magnet & II & 31.3 & 20.5 \\
& III & 59.85 & 11 \\
Double & I & 16.5 & 10 \\
magnet & II & 23.8 & 22.5 \\
& III & 30.4 & 18 \\
\hline
\end{tabular}

As shown in Figure 5, the highest amplitude at each magnet position occurs at different frequency. Position I, III and no magnet demonstrate quite similar amplitude but not at position II.

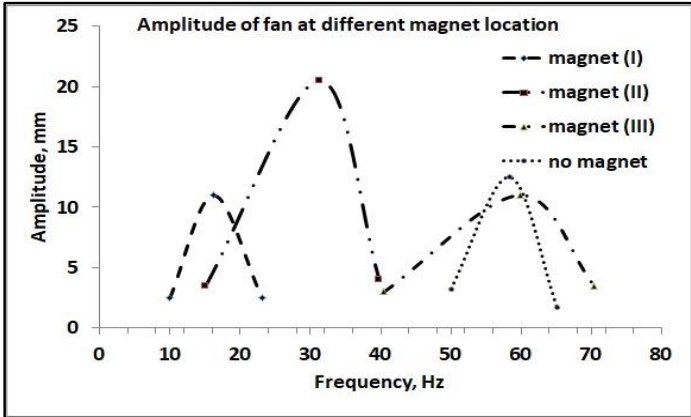

Figure 5 Amplitude of Fan at Different Magnet Position

The greatest amplitude achieved at position II is due to the moment of inertia for the fan is applied at the point. Moment of inertia is at lowest value at a point on the axis of rotation. Position II oscillates at largest amplitude at $20.5 \mathrm{~mm}$ at resonant frequency of $31.3 \mathrm{~Hz}$. Larger amplitude means more area is covered under the piezoelectric fan while frequency will determine the speed of the airflow caused by the oscillation of the fan.

\subsection{Single Magnet vs Dual Magnets on a Piezoelectric Fan}

By applying location II to put the magnet as additional mass, the amplitude readings were taken for single magnet and dual magnet at one piezoelectric fan to figure out which will give better amplitude. From Figure 6, it can be seen that dual magnets able to oscillate the fan larger than single magnet. 


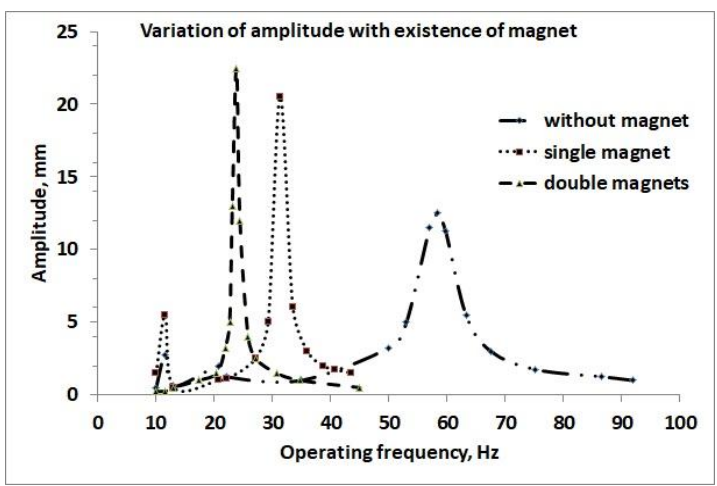

Figure 6. Variation of Amplitude with Existence of Magnet

It is important to figure out whether single or dual magnet will give the best amplitude result since more magnets means more repulsive force to drive other adjacent passive fan to enhance total amplitude in the integrated multiple piezoelectric fan and magnetic fans.

\subsection{Array vs Radial Orientation of Dual Fans}

In order to improve total amplitude of the system, dual fans have been used and arranged in array and radial orientation. By placing two magnets on each fan, the amplitude of each piezoelectric fan and passive fan are recorded at different distance between magnets which are $15 \mathrm{~mm}, 30 \mathrm{~mm}$ and $40 \mathrm{~mm}$. At the same distance between magnets, the results of different orientation are recorded as shown in Figure 7.

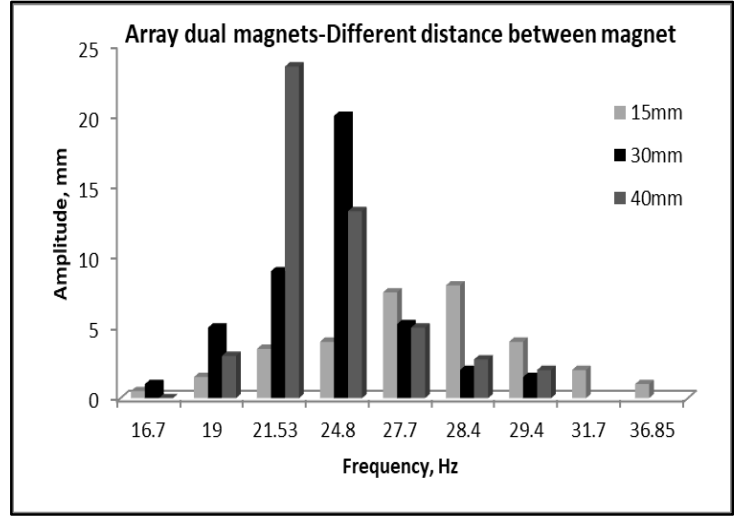

(a)

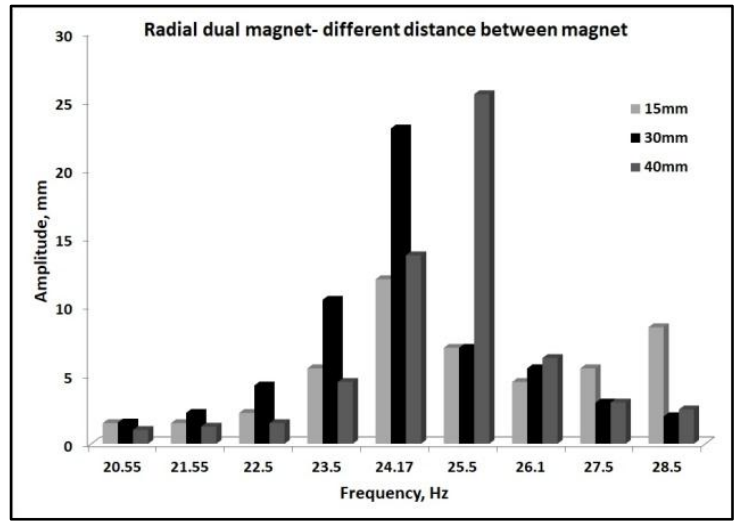

(b)

Figure 7. Total Amplitude of Dual Magnetic Fans at Different Distance between Magnets

Each case shows that radial orientation gives better result than array orientation. Distance between magnets should be selected in such a way that the amplitude produced by magnetic fan is not too far from the driving fan. Figure 8 shown the amplitude difference between piezoelectric fan and passive fan. 


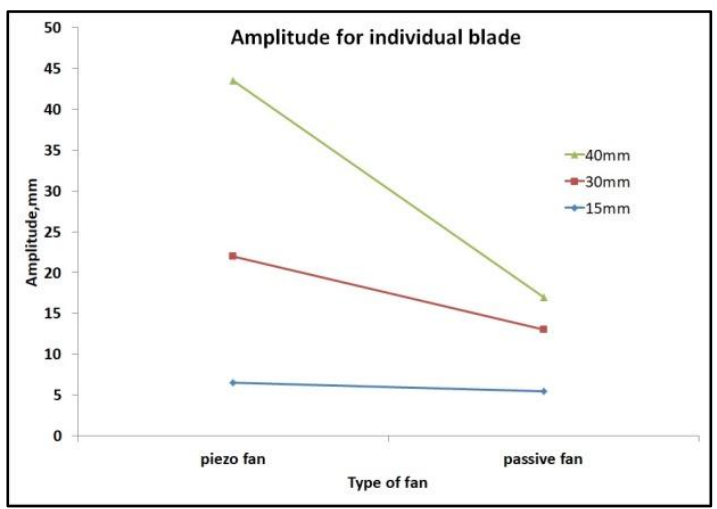

Figure 8. The Amplitude Difference between Piezoelectric Fan and Passive Fan

This is to stabilize the repulsive force delivered between the magnets and the magnetic fan oscillates evenly with the piezoelectric fan. Therefore, the magnets should be placed at a range of distance $15 \mathrm{~mm}-$ $30 \mathrm{~mm}$ to generate maximum total amplitude for dual fans (Figure 9).

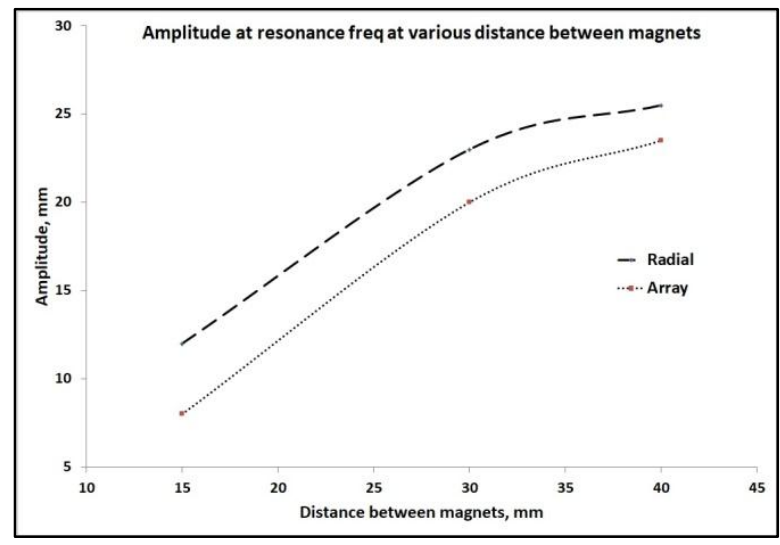

Figure 9. Total Amplitude Gained Different Fan Orientation

In Figure 9, at any distance between magnets, radial orientation consistently gives better amplitude by $25 \%$ compared to array orientation.

Enhancement in total amplitude applied in DPF shows positive improvement in total heat rejected due to the disturbance of air flow caused by the oscillation of the fan. Figure 10 has shown the rate of cooling at natural convection and forced convection by piezoelectric fan. Sudden drop of temperature shows that piezoelectric fan has high potential to enhance cooling system. 


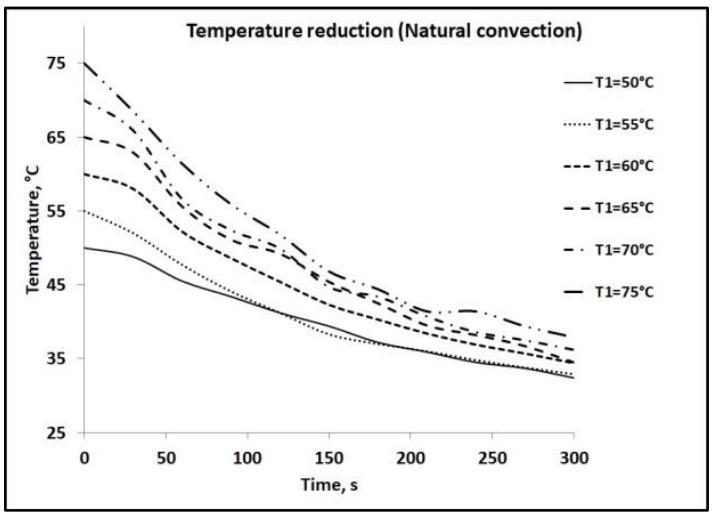

(a)

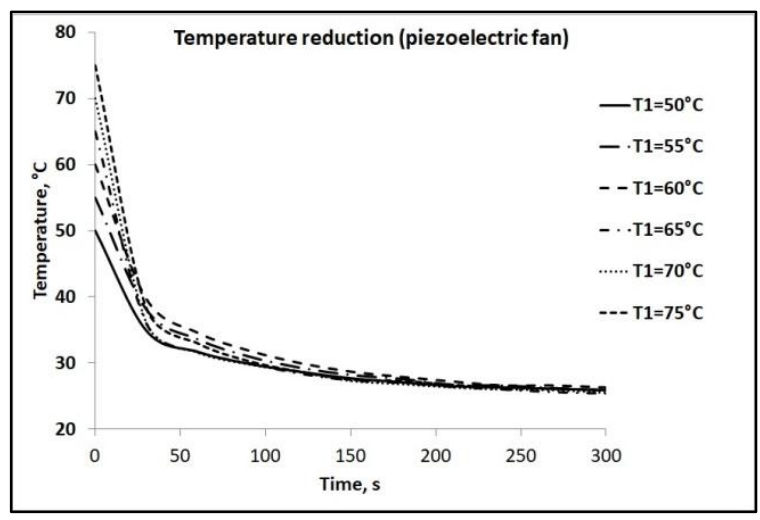

(b)

Figure 10. Cooling Rate of Natural Convection and dual Piezoelectric Cooling

This kind of system has a good enhancement in reducing power consumption while increasing the total amplitude to reduce the heat in the area. As shown in Figure 11, single fan with dual magnet located in the middle of the blade consumed the least power while producing highest total amplitude. Dual fans will definitely enhance the total amplitude without increasing the power consumption because no added piezoelectric required but only passive fans with magnets.

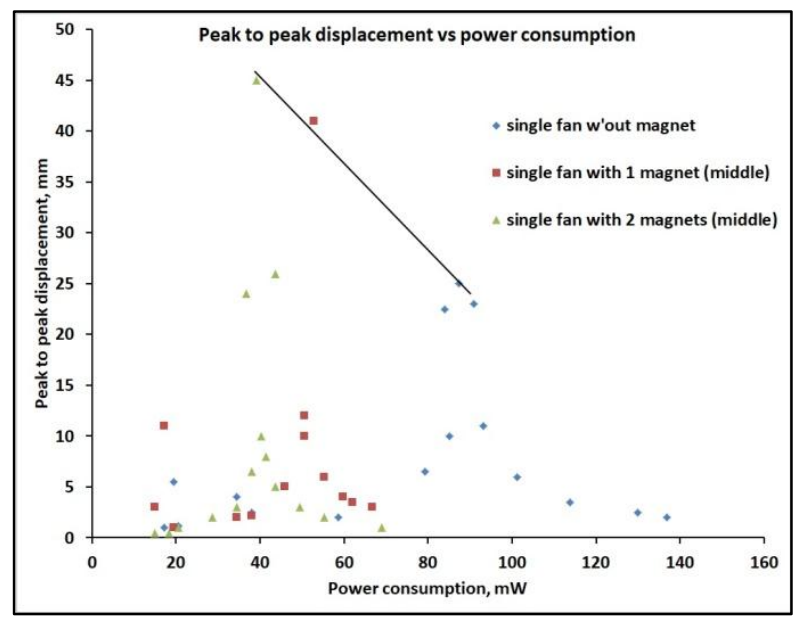

Figure 11. Peak to Peak Displacement vs Power Consumption at Different Number of Magnets

For measurement in heat transfer coefficient and thermal performance, the result is not so significant due to the comparison is made among single fan only. However, based on the good result in total amplitude and temperature drop recorded, heat transfer coefficient should be increasing and thermal resistance is reducing as parallel to the result obtained for temperature drop. Figure 12 shown thermal resistance vs power consumption at different magnet location. Figure 13 shown thermal resistance vs power consumption at different magnet location 


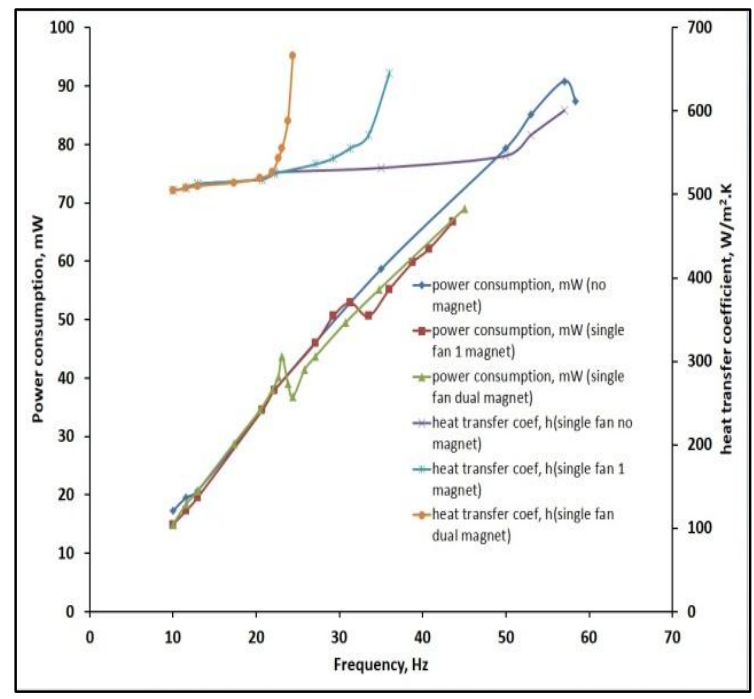

Figure 12. Thermal Resistance vs Power Consumption at Different Magnet Location

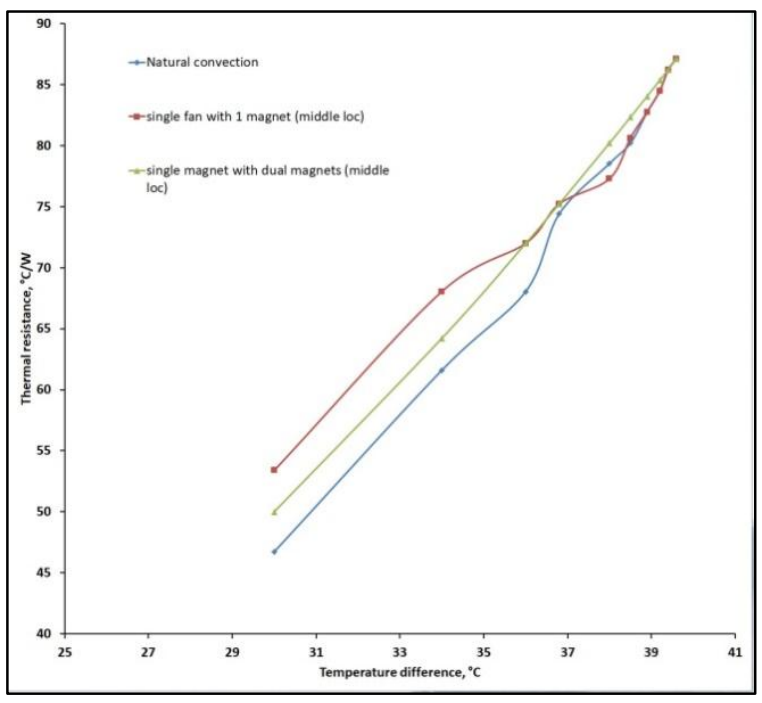

Figure 13. Thermal Resistance Vs Power Consumption At Different Magnet Location

\section{CONCLUSION}

Results show that the magnet at middle location of extensive blade with double magnets generate the largest amplitude, $80 \%$ better than fan without magnet and for dual integrated piezoelectric fan with magnetic fan, radial orientation gives better result by $25 \%$. By increasing the total amplitude, it shows a good agreement for positive heat transfer improvement compared to natural convection. For future works, the optimization of the magnet should be translated into enhancement in heat transfer coefficient and power consumption to be compared among piezoelectric fan with and without magnet, as well as between array and radial orientation.

\section{ACKNOWLEDGEMENTS}

The author would like to acknowledge Government of Malaysia under Fundamental Research Grant Scheme (FRGS) Vote R.K.130000.7840.4F850 under Ministry of High Education (MOHE) for financial support and Universiti Teknologi Malaysia for facilities and technical supports throughout the course of this research.

\section{REFERENCES}

[1] T. Açıkalın, I. Sauciuc, and S. V. Garimella, "Piezoelectric Actuators for Low-Form-Factor Electronics Cooling," Heat Transf. Summer Conf., no. San Francisco, California, USA, pp. 439-443, 2005.

[2] M. Kimber, K. Suzuki, N. Kitsunai, K. Seki, and S. V. Garimella, "Pressure and Flow Rate Performance of Piezoelectric Fans," IEEE Trans. Components Packag. Technol., vol. 32, no. 4, pp. 766-775, 2009.

[3] R. K. B. Schacht, A. Hausdorf, B. Wunderle, S. Rzepka, and B. Michel, "Efficiency Optimization for a Frictionless Air Flow Blade Fan - Design Study," in 14th IEEE ITHERM Conference, 2014, pp. 1019-1026.

[4] L. Léal, M. Miscevic, P. Lavieille, M. Amokrane, F. Pigache, F. Topin, and B. Nogarède, "An overview of heat transfer enhancement methods and new perspectives : Focus on active methods using electroactive materials," Int. $J$. Heat Mass Transf., vol. 61, pp. 505-524, 2013.

[5] M. Toda, "Theory of air flow generation by a resonant type PVF2 bimorph cantilever vibrator," Ferroelectrics, vol. 22, no. 1, pp. 911-918, 1978.

[6] J. Yang, Analysis of Piezoelectric Devices. 2006.

[7] S. Kakac, H. Yuncu, and K. Hijikata, Cooling of Electronic Systems. 1994.

[8] Y. Cao and X. B. Chen, "A Survey of Modeling and Control Issues for Piezo-electric Actuators," J. Dyn. Syst. Meas. Control, vol. 137, no. 1, p. 14001, 2014.

[9] R. V.Dukkipati, Solving Vibration Analysis Problems Using Matlab. 2007.

[10] S. Canfield and M. Frecker, "Topology optimization of compliant mechanical amplifiers for piezoelectric actuators," Struct. Multidiscip. Optim., vol. 20, no. 4, pp. 269-279, 2000.

[11] K. Tseng, M. Mochizuki, and K. Mashiko, "Piezo Fan for Thermal Management of Electronics," Fujikura Tech. Rev., pp. 39-43, 2010. 
[12] M. S. A. A. Z.M. Fairuz, S.F. Sufian, M.Z. Abdullah, M. Zubair, "Effect of piezoelectric fan mode shape on the heat transfer characteristics," Int. Commun. Heat Mass Transf., vol. 52, pp. 140-151, 2014.

[13] T. Yeom, T. W. Simon, L. Huang, M. T. North, and T. Cui, "Piezoelectric translational agitate on for enhancing forced-convection channel-flow heat transfer," Int. J. Heat Mass Transf., vol. 55, no. 25-26, pp. 7398-7409, 2012.

[14] T. Acikalin, S. M. Wait, S. V Garimella, and A. Raman, "Experimental investigation of the thermal performance of piezoelectric fans," Heat Transf. Eng., vol. 25, no. 1, pp. 4-14, 2004.

[15] H. C. Su, C. L. Liu, T. J. Pan, and H. K. Ma, "Investigation of a Multiple-Vibrating Fan System for Electronics Cooling," in 29th IEEE SEMI-THERM Symposium, 2013, vol. 1, pp. 110-116.

[16] H. K. Ma, H. C. Su, and W. F. Luo, "Investigation of a piezoelectric fan cooling system with multiple magnetic fans," Sensors Actuators, A Phys., vol. 189, pp. 356-363, 2013.

[17] J. C. Shyu and J. Z. Syu, "Plate-fin array cooling using a finger-like piezoelectric fan," Appl. Therm. Eng., vol. 62, no. 2, pp. 573-580, 2014.

[18] M. K. Abdullah, N. C. Ismail, M. A. Mujeebu, M. Z. Abdullah, K. A. Ahmad, M. Husaini, and M. N. A. Hamid, "Optimum tip gap and orientation of multi-piezofan for heat transfer enhancement of finned heat sink in microelectronic cooling," Int. J. Heat Mass Transf., vol. 55, pp. 5514-5525, 2012.

[19] Z. Syafiqah, Y.M. Irwan, N.A.M. Amin, M. Irwanto, W.Z. Leow, and A.R. Amelia. Thermal and Electrical Study for PV Panel with Cooling. Indonesian Journal of Electrical Engineering and Computer Science (IJEECS), 7(2), 492-499, 2017. 